\title{
La littérature contemporaine aux prises avec le mensonge et la mauvaise foi
}

\section{Maxime Decout et Jochen Mecke}

\section{(2) OpenEdition \\ Journals}

Édition électronique

URL : https://journals.openedition.org/fixxion/295

DOI : $10.4000 /$ fixxion.295

ISSN : 2295-9106

Éditeur

Ghent University

Référence électronique

Maxime Decout et Jochen Mecke, « La littérature contemporaine aux prises avec le mensonge et la mauvaise foi », Revue critique de fixxion française contemporaine [En ligne], 22 | 2021, mis en ligne le 15 juin 2021, consulté le 17 février 2022. URL : http://journals.openedition.org/fixxion/295 ; DOI : https:// doi.org/10.4000/fixxion.295

Ce document a été généré automatiquement le 17 février 2022.

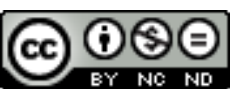

Les contenus de la Revue critique de fixxion française contemporaine sont mis à disposition selon les termes de la licence Creative Commons Attribution - Pas d'Utilisation Commerciale - Pas de Modification 4.0 International. 


\title{
La littérature contemporaine aux prises avec le mensonge et la mauvaise foi
}

\author{
Maxime Decout et Jochen Mecke
}

1 Entre mensonge et mauvaise foi, la littérature est en terrain connu. Tout le monde sait cependant qu'une fiction ne peut pas réellement mentir. Le lecteur, à moins de s'appeler Don Quichotte, n'ignore pas que l'histoire est fictive. C'est pourquoi un numéro de Fixxion sur le mensonge et la mauvaise foi pourrait étonner et presque prendre des allures d'oxymore, puisque, en soi, la littérature fictionnelle est capable de tout sauf de mentir. Cette perspective pourrait paraître plus surprenante encore à propos de la littérature contemporaine dont le retour au réel n'est plus à démontrer' ${ }^{1}$. Cette transitivité retrouvée semble exclure a priori, ou tout au moins minorer, la part de jeu que les textes puisent dans le mensonge et la mauvaise foi. Et pourtant, entre le document, l'archive et la non-fiction, les œuvres ne manifestent-elles pas ostensiblement leur désir d'authenticité ? En multipliant les signes d'une transparence dans laquelle le réel primerait sur l'écriture, la littérature ne réagit-elle pas au soupçon de mensonge qui plane sur elle? D'autant mieux que, malgré l'omniprésence de cette littérature du réel qui donne l'impression d'être débarrassée de pièges et de fraudes, le mensonge et la mauvaise foi n'ont pas dit leur dernier mot et restent toujours éminemment productifs, comme sous la plume de Pierre Senges, Tanguy Viel, Antoine Bello ou Éric Chevillard.

2 Pour toutes ces raisons, une étude de la littérature contemporaine à travers le prisme du mensonge et de la mauvaise foi pourrait se légitimer en répondant à deux questions. La première est liée à l'apport épistémique de ces catégories - mensonge et mauvaise foi - pour l'analyse de la littérature contemporaine et de ses préoccupations esthétiques; la seconde s'effectue à rebours, et porte sur ce que la littérature contemporaine peut, quant à elle, apporter à la compréhension du mensonge et de la mauvaise foi. 


\section{Dans la caverne de Platon : le mythe de la condamnation des poètes menteurs}

3 Si malgré toutes les objections évidentes, les critiques contemporaines parlent du mensonge sous le jour du reproche, elles évoquent en fait une condamnation qui accompagne la littérature depuis l'Antiquité. On en trouve l'origine chez Platon, ou plutôt dans la vulgate d'une certaine réception platonicienne, d'après laquelle Platon aurait banni les poètes de l'État idéal pour avoir menti - c'est-à-dire pour avoir inventé des histoires fictionnelles. Or un examen attentif de La république montre que les raisons de cet anathème sont plus ambiguës qu'il n'y parait, celui-ci étant justifié de deux manières très différentes et dont aucune ne se réfère à la notion de fiction. Le premier argument avancé par le personnage de Socrate chez Platon résulte directement de l'ontologie platonicienne : puisque la vérité correspond à la réalité supérieure des Idées, le réel perceptible est - selon la célèbre allégorie de la caverne le reflet trompeur d'une réalité supérieure ${ }^{2}$. Par conséquent, poursuit Platon, les produits de l'art - et surtout de l'art réaliste - seraient affectés d'une double fausseté : reflets trompeurs d'un autre reflet trompeur. Ce premier "mensonge" est intrinsèque à toute forme d'art et ne saurait donc servir de chef d'accusation moral contre les poètes. Le deuxième argument à l'encontre de ces derniers relève en revanche du domaine de l'éthique, ou plus précisément de la pédagogie, car Socrate blâme les écrivains - et cela constitue l'élément décisif pour leur expulsion - en raison du portrait peu flatteur qu'ils livrent des dieux ou de héros comme Ulysse, prompt à tromper, mentir et louer l'ivrognerie. Socrate prononce alors son verdict: les poètes seront chassés de la République car ils blessent la religion et donnent de mauvais exemples à la jeunesse. "Ces raisons, dit-il, nous obligent à ne plus permettre de pareilles fictions, de peur qu'elles ne produisent dans la jeunesse une malheureuse facilité à commettre le crime"3. Socrate bannit donc les poètes non pas parce qu'ils créent des fictions mais parce qu'ils dressent un tableau trop "humain" et "réaliste" des dieux et des héros. Quant au mensonge, Socrate va jusqu'à le justifier expressis verbis et en faire un outil légitime s'il permet de fournir à la jeunesse des modèles positifs ${ }^{4}$. Voilà qui autorise Socrate à instaurer lui-même ce qu'il appelle un "noble mensonge", qui garantirait la stabilité de la structure hiérarchique de l'État et que les éducateurs devraient relayer auprès des enfants 5 .

4 Il est regrettable que les véritables motifs de cette mise au ban des poètes aient été réduits, au cours de l'histoire littéraire, à l'idée d'un mensonge qui tiendrait avant tout à la nature fictionnelle des œuvres. Le succès de cette vulgate est d'autant plus déroutant qu'un rapide regard sur les notions de fiction et de mensonge montre bien que la fiction en soi n'est pas susceptible d'être un mensonge. Pour le comprendre, il convient de revenir aux caractéristiques définitoires du mensonge, à savoir qu'il s'agit d'abord d'une divergence entre conviction ou sentiments d'une part et expression de l'autre (1), ensuite d'une dissimulation de cette divergence (2), laquelle sert enfin des objectifs eux-mêmes camouflés (3). Au vu de ces critères, il va de soi qu'une fiction littéraire, sauf à les mettre en pratique, ne saurait être considérée comme relevant $a$ priori du mensonge ${ }^{6}$. Au contraire, la fiction dévoile le plus souvent d'emblée son statut d'histoire inventée, ne serait-ce que par certaines dénominations génériques, comme "roman" ou "théâtre". Elle rend de fait inopérante la dissociation censément déguisée entre conviction et opinion. Le mensonge, dénoncé comme tel, n'en est plus un. 
Pareille situation vaut également pour l'ensemble des marqueurs de littérarité, comme le passé simple ou les figures rhétoriques, qui peuvent être tenus pour des signes révélant au destinataire du message que le texte appartient à un ordre du discours singulier, qui n'est pas à prendre au pied de la lettre mais où prévaut la polysémie. Friedrich Nietzsche a du reste insisté sur la relation antinomique de l'art et du mensonge en affirmant : "Kunst behandelt den Schein als Schein, will also gerade nicht täuschen, ist wahr"7. Si l'art et la littérature font donc volontairement étalage du caractère fictif de leurs créations, si tous deux dénoncent ainsi leur propre mensonge, ils ne sauraient point mentir - du moins au sens propre. Et il n'est pas insignifiant, dans cette perspective, que l'histoire du roman moderne commence sous l'égide de Cervantès avec un lecteur incapable de décoder les signaux du mensonge et qui prend les hauts faits romanesques des livres de chevalerie au sens propre. Contrairement à ce type de méprise, aucun lecteur n'est dupé par ce genre de supercherie. C'est sans doute pour cette raison que la littérature est devenue un véritable observatoire des mensonges et de tout type de tricheurs, hypocrites, imposteurs ou intrigants.

\section{La littérature contemporaine : un observatoire du mensonge et de la mauvaise foi ?}

Depuis ses origines, la littérature s'est abondamment penchée sur les mensonges et la mauvaise foi qui caractérisent nos existences et nos sociétés. L'époque contemporaine ne fait pas exception. Les menteurs, hypocrites et imposteurs sont légion. Qu'on pense seulement à Mentir ou Fraudeur de Savitskaya, Le troisième mensonge d'Agotha Kristof, L'adversaire d'Emmanuel Carrère, L'imposteur de Javier Cercas, Un secret de Philippe Grimbert, L'épave ou Pas dupe d'Yves Ravey, Le faussaire de Yasushi Inoué, Mensonge(s) de Patrick Delétang, Impostures de John Banville, Imposture de Vila-Matas, ou encore aux nombreux arnaqueurs, mythomanes et fabulateurs qui peuplent les récits de Tanguy Viel (Le Black Note, Paris-Brest, Insoupçonnable...). Les titres de ces récits sont signifiants de plus d'une manière. Ils témoignent d'abord de la fascination que les fraudes et la tricherie continuent à exercer sur la littérature. Mais ils signalent aussi la variété des formes que ces dernières revêtent. Car la littérature contemporaine parcourt à loisir un vaste répertoire d'attitudes allant de la mauvaise foi à l'imposture. Plus encore : elle tend à diversifier, voire à réinventer, les conduites de mensonge et de mauvaise foi, en en démultipliant les possibles.

7 Face à un tel panorama, on aurait vite fait de conclure que la littérature contemporaine se singularise par cette attirance pour toutes les formes du mensonge. Or la question qui se pose est bien de savoir si la représentation des menteurs et de leurs boniments diffère fondamentalement de celle qui prévalait au $\mathrm{XX}^{\mathrm{e}}$ siècle, et si les textes en question relèvent de nouvelles attitudes dans le traitement esthétique et moral du mensonge. Il faut d'abord reconnaître que, sur ce plan, la littérature contemporaine s'inscrit dans une certaine continuité avec le $\mathrm{XX}^{\mathrm{e}}$ siècle qui ne fut pas avare en escrocs, bonimenteurs et autres charlatans. Elle sonde cependant avec acuité les mutations les plus récentes de nos sociétés, marquées par le complotisme, les fake news, l'ère de la post-vérité et le story-telling, autant d'éléments qui pénètrent avec force dans les textes, comme par exemple dans la trilogie d'Antoine Bello (Les falsificateurs, Les éclaireurs et Les producteurs) ou dans La montagne de Minuit de Jean-Marie Blas de Roblès. 


\section{Une littérature menteuse?}

Penser la littérature à travers le mensonge et la mauvaise foi ne peut toutefois se borner à observer la façon dont elle les ausculte à travers ses intrigues et ses personnages. Car bien des œuvres, séduites par le pouvoir des boniments, affrontent la délicate question de savoir comment faire mentir une fiction qui n'est pas en ellemême un mensonge. Si les quelques exemples cités ci-dessus montrent que la littérature est l'une des premières observatrices des pratiques mensongères, elle a également appris, du moins depuis l'époque moderne, à mentir - dans un sens toutefois bien différent du simple fait de raconter des histoires inventées. On peut évidemment penser en premier lieu aux mystifications, apocryphes, et pseudonymes ${ }^{8}$ qui, après Machado, Pessoa et Gary, trouvent un second souffle de nos jours sous la plume de Jean-Benoit Puech ou Volodine. Mais le plus souvent l'importation du mensonge dans l'univers littéraire se fait de manière plus discrète en tentant non pas de transformer la fiction en mensonge mais au moins de lui en donner les principaux traits, à partir de quelques-unes de ses caractéristiques. C'est ainsi qu'un mensonge mis en scène (intradiégétique) peut non pas devenir mais faire croire à l'existence d'un mensonge destiné au lecteur (extradiégétique). Or c'est principalement avec l'instance narrative que cette percée du mensonge dans la fiction est opérée. L'un des moyens les plus efficaces pour mimer ce passage d'un mensonge intradiégétique à un mensonge extradiégétique est en effet d'agir sur celui qui se tient à la charnière du monde du lecteur et du monde du livre, le narrateur.

C'est certainement avec le narrateur homodiégétique, en première personne, que la suppression fantasmée des frontières entre mensonge dans le texte et mensonge au lecteur revêt ses formes les plus spectaculaires. Ce procédé est déjà à l'œuvre dans le premier roman picaresque, Lazarillo de Tormes, dans lequel le héros, qui est en même temps le narrateur, ment non seulement en tant que personnage, à l'intérieur de l'histoire, mais aussi en tant que narrateur à l'échelle du roman, en induisant le lecteur en erreur. Nous sommes donc confrontés à un mensonge au deuxième degré, c'est-àdire un mensonge qui s'effectue au sein de ce "mensonge" avoué, et donc invalidé, qu'est la fiction. Dans la plupart des cas, ces mensonges au deuxième degré sont relatifs à un narrateur autodiégétique, un procédé qui joue souvent sur le double statut du narrateur-personnage. Quant à la littérature contemporaine du XXe siècle et du XXI siècle, elle a évidemment continué à explorer cette veine d'un narrateur "non fiable" ou menteur 9 . Plus exactement, les récits approfondissent une série d'attitudes narratoriales, qui vont du narrateur non fiable, d'un point de vue factuel, au narrateur indigne de confiance, du point de vue de ses valeurs et de l'idéologie ${ }^{10}$.

Pour n'en citer que quelques exemples : dans La symphonie pastorale de Gide, le pasteur relate dans son journal intime l'arrivée d'une jeune orpheline aveugle, Gertrude, qu'il se propose d'éduquer et dont il veut élever l'âme. Le récit autodiégétique attribue toujours les actions du pasteur aux motifs les plus nobles et les plus altruistes, comme lorsqu'il empêche son fils Jacques d'épouser Gertrude. Mais la vérité finit par éclater : le pasteur agit en raison de sa passion amoureuse pour sa pupille. Gide conçoit de la sorte un cas où le protagoniste ment à ses enfants et à sa famille, mais où le narrateur ment également à son lecteur. Toutefois, dans le cas de La symphonie pastorale, le statut du mensonge est équivoque, puisqu'il appartient en même temps à l'histoire et au discours narratif. En vertu de la forme du journal intime, il reste difficile de décider si le pasteur 
se ment simplement à lui-même, en toute mauvaise foi, ce qui relèverait de l'histoire racontée, ou bien s'il ment également au lecteur, ce qui relèverait du discours narratif. Le roman de Gide puise une partie de son intérêt esthétique dans cette ambiguïté fondamentale que l'on retrouve dans la fiabilité douteuse de l'instance narrative de Molloy de Beckett, dans les confessions de Clamence truffées de mensonges et de mauvaise foi dans La chute de Camus, ou dans les contradictions du narrateur Jacques Revel dans L'emploi du temps de Butor, qui poussent le lecteur et le héros lui-même à se méfier de la narration.

11 Là encore, la littérature contemporaine emboîte le pas à celle qui l'a précédée. Après $L a$ symphonie pastorale de Gide, Molloy et L'innommable de Beckett, La chute de Camus, La méprise de Nabokov, Le bavard de Des Forêts, on ne peut manquer de remarquer la non fiabilité, sur des plans très divers, des narrateurs du Black Note de Tanguy Viel, Pas dupe d'Yves Ravey, La danse du fumiste de Paul Emond, Veuves au maquillage de Pierre Senges, L'auteur et moi, Démolir Nizard et L'ouvre posthume de Thomas Pilaster de Chevillard ou des Bienveillantes de Littell où Max von der Aue, un ancien officier SS qui a participé de manière active à l'extermination des Juifs, ment autant sur le plan épistémologique que sur le plan idéologique. À ce titre, c'est la catégorie de narrateur non fiable qui est régulièrement mobilisée.

12 Tous ces cas de figure ne sont bien entendu pas équivalents et introduisent de subtils dosages dans la mauvaise foi et le mensonge des instances narratives et des personnages. Si cette veine, on le voit, est toujours vive, elle paraît toutefois dans l'ensemble moins fréquente et surtout moins virtuose. Les coups de théâtre qui dévoilent la supercherie, comme dans La chute, La méprise et Le bavard, sont plus rares, les œuvres privilégiant plutôt l'ambivalence et l'incertitude propres à une attitude où la mauvaise foi et la désinvolture l'emportent sur le mensonge avéré. Les narrateurs de Veuves au maquillage ou de Démolir Nizard par exemple sont des individus peu fréquentables, que le lecteur suspecte à longueur de pages sans avoir de certitude sur leurs mensonges puisque ceux-ci semblent davantage justifiés par une disposition d'esprit du personnage que par un objectif sournois qu'il cacherait au lecteur. C'est, le plus souvent, la dimension ludique et ambiguë qui est mise en avant, appelant le lecteur à une vigilance accrue face au texte et jetant le doute sur ses interprétations.

Minorant la part prise par la virtuosité d'un narrateur homodiégétique trompeur, la littérature contemporaine s'est tournée vers des formes moins flagrantes du mensonge, souvent plus proches d'une mauvaise foi larvée. Lorsque le narrateur ne fait pas partie des personnages de l'histoire, la possibilité de mentir au second degré est en effet tout autant manifeste. Ce narrateur hétérodiégétique a toute marge de manœuvre pour falsifier les choses grâce à son omniscience. Là aussi, la littérature contemporaine n'hésite pas à reprendre les entorses à la fiabilité de ce narrateur qui ponctuent certains œuvres du $\mathrm{XX}^{\mathrm{e}}$ siècle, en particulier chez les auteurs du Nouveau Roman. Robbe-Grillet a largement fait fructifier ces infractions, comme dans Le voyeur ou Projet pour une révolution à New York pour s'en tenir à deux exemples. Dans ces récits, les mensonges du narrateur hétérodiégétique, qui vont de la rétention d'information à la déformation des faits, ne sont le plus souvent que soupçonnés et demeurent impossibles à avérer complètement. Ils créent un malaise permanent et déroutent le lecteur, tout en désignant le roman lui-même dans une démarche réflexive qui dénonce l'inauthenticité et les leurres de toute fiction. 
Le lecteur de Jean Echenoz n'est pour sa part ni inquiété ni égaré continument quand le romancier s'empare du procédé de manière ludique, aussi bien dans Un an, Envoyée spéciale que Je m'en vais. Racontant les aventures d'un galeriste appelé Ferrer, ce roman est issu d'un narrateur hétérodiégétique dont la présence est sensible tout au long du texte à travers ses nombreux commentaires, évaluations ou opinions. Comme c'est souvent le cas chez Echenoz, ce narrateur omniscient et extérieur à l'histoire prend les traits d'une figure presque sympathique, qui guide le lecteur avec bienveillance. C'est lui qui nous fait assister à la mort de Delahaye, l'assistant de Ferrer, et qui, ensuite, nous montre les agissements d'un certain Baumgartner qui a dérobé les œuvres en possession du galeriste. Or, nous ne le découvrons que très tard, Delahaye, censément mort, n'est en fait rien d'autre que Baumgartner lui-même. Notre sympathique narrateur nous a donc menti. Il connaissait l'identité des deux personnages et a choisi délibérément de ne pas la divulguer. Mais ce mensonge reste extrêmement circonscrit et n'affecte pas d'autres éléments de l'histoire. Il ne plonge pas l'ensemble du récit dans l'équivoque, n'immerge pas le lecteur dans un monde étrange où les assurances sont sapées par le mensonge. C'est sur un mode mineur, en tant qu'infraction ponctuelle et fantaisiste, presque sous forme de clin d'œil, que ce genre de transgressions opère.

La particularité de ce procédé apparaît clairement si nous le comparons aux changements de narrateurs dans le Nouveau Roman. On peut évoquer Claude Simon qui fait alterner dans La route des Flandres des instances narratives homodiégétique et hétérodiégétique et désigne le personnage central de Georges tour à tour par "Je" ou par "Il", ou encore Le ravissement de Lol V. Stein, dans lequel Marguerite Duras modifie le statut du narrateur au milieu du texte. Dans les deux cas, ces transformations rompent ouvertement avec un certain code du roman conventionnel. Si Robbe-Grillet, Claude Simon ou Marguerite Duras entendaient pour leur part miner la prétention du narrateur à l'omniscience et le confort d'un récit vraisemblable à la Balzac, ce n'est plus exactement le cas dans la littérature contemporaine. Il ne s'agit pas uniquement de donner raison aux doutes du lecteur à "l'ère du soupçon", de faire la lumière sur un "mensonge romanesque" - qui consisterait à faire croire à la possibilité d'un narrateur capable de tout savoir - au nom d'une vérité plus authentique : celle de la limitation de la perspective et du savoir. Alors que le Nouveau Roman rompt avec ces conventions jugées fallacieuses et mensongères pour les remplacer par un autre système narratif plus authentique, des romanciers contemporains tels que Deville ou Echenoz montrent que ces conventions peuvent être utilisées pour créer de véritables mensonges romanesques. Tandis que le Nouveau Roman adopte une position extérieure au système narratif usuel pour le déconstruire, le roman contemporain le sape pour ainsi dire de l'intérieur. Les deux procédés partagent un souci d'exposer certains éléments du système narratif, mais à la différence du Nouveau Roman, le "roman nouveau" produit sciemment un mensonge narratif qui induit le lecteur en erreur, tout en respectant apparemment le code narratif traditionnel que le Nouveau Roman stigmatise. Lorsque le narrateur d'Echenoz désigne la même personne par des noms différents, tout en connaissant leur identité, le texte ne rompt pas de manière explicite avec le code romanesque, comme le faisaient Simon, Duras, Butor ou Robbe-Grillet avant lui, mais il s'en sert pour tromper le lecteur. Echenoz mobilise le mensonge du narrateur en ne masquant pas son artificialité, comme s'il s'agissait d'un jeu propre au roman policier ou au roman feuilleton dans lesquels ce genre de dissimulation n'est pas rare - qu'on pense seulement au cas de Claude Frollo dans Notre Dame de Paris. Contrairement aux auteurs du Nouveau Roman, Echenoz doit respecter et même présupposer les 
conventions narratives pour que son mensonge puisse fonctionner. En résulte un mensonge spécifiquement littéraire en tant qu'il recourt précisément aux instruments et aux codes attendus pour tromper le lecteur. Nous faisons pour ainsi dire l'expérience concrète d'un mensonge que le Nouveau Roman, lui, ne faisait que dénoncer.

\section{Vers une remise en question de la compréhension habituelle du mensonge et de la mauvaise foi}

16 Par sa manière de réinterroger le mensonge et la mauvaise foi, la littérature des $\mathrm{XX}^{\mathrm{e}}$ et $\mathrm{XXI}^{\mathrm{e}}$ siècles contribue donc à envisager sous un jour différent certains présupposés de la conception convenue du mensonge, une remise en cause qui concerne surtout les deux termes les plus importants de notre définition, à savoir la conviction et l'expression.

Le Nouveau Roman l'a clairement montré, notamment avec ce que Nathalie Sarraute a appelé la "sous-conversation" : la conscience d'un personnage ne se laisse pas réduire à une seule voix, mais repose sur un entremêlement de voix disparates réunies en un polylogue intérieur, perpétuel et souterrain. De cette manière, l'unité de la conscience individuelle se disloque au gré de voix éparses, qui, dans la plupart des cas, sont en conflit les unes avec les autres. Or cette dissolution de l'unité de la conscience individuelle, parce qu'elle ne permet plus de déterminer la conviction véritable du personnage, rend du même coup caduque toute échelle de mesure pour déterminer s'il y a ou non mensonge. De plus, l'écriture expérimentale de Nathalie Sarraute s'attaque également au deuxième terme de l'équation de notre définition du mensonge, c'est-àdire à l'expression, car ses romans scrutent les lieux communs de la conversation quotidienne.

C'est pourquoi nous sommes aussi invités à considérer les mensonges du personnage, et par contrecoup les œuvres qui les mettent en scène, à l'aune d'une notion plus ambivalente : la mauvaise foi ${ }^{11}$. Du moins la mauvaise foi telle que Sartre la théorie dans L'être et le néant. Celui-ci part du constat que la conscience cherche toujours à coïncider avec elle-même bien que cela soit impossible. Lorsque le sujet se rend compte de cette inadéquation et la refuse, il est amené à la maquiller. Il bascule alors dans la mauvaise foi, conduite par laquelle il tente de masquer et de se masquer qu'il ne coïncide jamais tout à fait avec ce qu'il est. La mauvaise foi serait dès lors cette attitude où un individu nie ce qu'il est en fonction de ce qu'il croit, veut ou doit être, à ses yeux ou aux yeux des autres, et qu'il n'est pas. De sorte que le sujet en proie à la mauvaise foi est toujours ce qu'il n'est pas et n'est pas ce qu'il est. Conçue de la sorte, la mauvaise foi n'est donc pas évaluable en regard de la seule vérité. Elle ouvre le sujet à des voix multiples qui traversent la conscience et elle place le mensonge hors d'une conviction de départ, d'un projet ou d'objectifs clairement identifiables.

19 Dans le même temps, Nathalie Sarraute a signalé comment, jusque dans la conscience, interviennent des formes inauthentiques de l'expression, qui ne sont pas sans affinité avec l'inauthenticité propre à la mauvaise foi. Car nous pouvons mentir sans le vouloir, nous pouvons sombrer dans la mauvaise foi inconsciemment, situation qui, en dehors de l'unité de la conscience, met donc en question une deuxième présupposition de toute théorie du mensonge selon laquelle nous avons toujours à notre disposition les mots propres à traduire nos convictions et nos sentiments. On peut remarquer la justesse des observations sarrautiennes dans la conversation de tous les jours, où 
certaines expressions ou images peuvent nous sembler "fausses", inadéquates ou trompeuses, sans que personne n'ait pour autant eu l'intention de mentir. Des formules comme "les heures sombres de l'histoire", "l'amitié entre les peuples", "nos frères et sœurs en Lybie" peuvent être perçues comme des mensonges en ce sens. Dans ce cas, l'écart entre opinion et expression provient de l'incapacité du locuteur ou bien de l'impossibilité générale de donner une expression adéquate à ce que l'on pense ou ressent. En montrant que nous pouvons mentir ou être affectés de mauvaise foi par défaut de termes plus appropriés pour exprimer nos pensées, la littérature s'attaque à la troisième présupposition de la conception habituelle du mensonge, à savoir l'idée qu'un mensonge est toujours intentionnel. La littérature, caractérisée par sa propension à sinuer dans les eaux troubles de l'ambiguïté, est très clairement le discours qui est le plus à même de représenter et d'ausculter ces états intermédiaires de l'être et de sa conscience.

Mais si la littérature moderne propose, comme nous l'avons vu, une critique du mensonge et de sa compréhension habituelle, si elle en fait le diagnostic, elle suggère aussi des remèdes, car elle se conçoit également comme une quête de formes d'expression qui puissent résoudre les défaillances et les lacunes du langage qui lui sont pourtant inhérentes. Dans Le planétarium Sarraute ne se contente pas de dénoncer les clichés et les lieux communs de la conversation, elle développe, par images et métaphores, des formes d'expression authentiques de ce qu'elle appelle les "tropismes", c'est-à-dire des "mouvements indéfinissables qui glissent très rapidement aux limites de notre conscience". Ceux-ci "sont à l'origine de nos gestes, de nos paroles, des sentiments que nous manifestons, que nous croyons éprouver et qu'il est possible de définir"12. De nouvelles formes narratives permettent d'explorer ces espaces plus profonds de l'être humain qui, jusque-là, étaient restés cachés. De fait, les tropismes ne peuvent être représentés par les moyens littéraires classiques, pas même par le monologue intérieur, car ils se soustraient aux lieux communs de la conversation et au langage en général. C'est pour cela que Sarraute cherche à les communiquer au lecteur par le biais d'images capables de proposer des significations équivalentes et d'éprouver des sensations analogues ${ }^{13}$. Et, en même temps qu'elle dénonce l'inauthenticité de la conversation, avec ses lieux communs, elle critique la forme du roman traditionnel, puisque la question du mensonge ne se pose pas seulement au niveau de l'histoire, mais investit de surcroît le niveau de la représentation littéraire. Le domaine de l'esthétique se trouve de la sorte intimement lié à celui du mensonge.

On peut dès lors interpréter la dynamique particulière de la littérature moderne comme une tentative de renouvellement esthétique permanent qui est motivée par la critique du caractère inauthentique des formes déjà acceptées et consacrées. Les œuvres ont ainsi développé un mensonge spécifiquement littéraire qui englobe différentes formes de ruptures avec ce qui peut être tenu pour l'impératif catégorique de la modernité : l'exigence d'authenticité. Dans un texte devenu célèbre, Vérité et mensonge au sens extra-moral, Friedrich Nietzsche a élaboré une conception du mensonge qui explique cette dynamique :

les vérités sont [...] des métaphores qui ont été usées et qui ont perdu leur force sensible, des pièces de monnaie qui ont perdu leur empreinte et qui entrent dès lors en considération, non plus comme pièces de monnaie, mais comme métal. [...] être véridique, cela signifie employer les métaphores usuelles; donc, en termes de morale, l'obligation de mentir selon une convention ferme, de mentir grégairement dans un style contraignant pour tous. L'homme oublie assurément qu'il en est ainsi 
en ce qui le concerne; il ment donc inconsciemment de la manière désignée et selon des coutumes centenaires - et, précisément grâce à cette inconscience et à cet oubli, il parvient au sentiment de la vérité. ${ }^{14}$ "force sensible" et leur authenticité, qui aboutirait à la création de nouvelles formes perçues comme plus véridiques et originales. Cette négation de la tradition romanesque se fait cependant sous le signe d'une continuité plus profonde, car elle se conçoit comme une perpétuation de l'esthétique moderne de l'authenticité. Comme le rappelle Alain Robbe-Grillet, "l'art doit être perpétuellement en rupture avec l'art qui le précède immédiatement" ${ }^{15}$. Les reproches que le Nouveau Roman adresse au roman conventionnel, jugé inauthentique, relèvent bien d'un mensonge esthétique compris comme moteur de l'évolution des œuvres. Toutefois, la forme spécifiquement moderne de l'authenticité n'est pas uniquement due à l'expression adéquate des convictions et des sentiments de l'auteur, mais aussi à son actualité, à sa relation intime et éphémère avec l'époque qui le voit naître. Comme le souligne Baudelaire dans Le peintre de la vie moderne "presque toute notre originalité vient de l'estampille que le temps imprime à nos sensations"16. Ainsi, les épigones ou les prosélytes d'un auteur ou d'un mouvement littéraire enfreignent une loi esthétique, car ils violent l'impératif catégorique de l'authenticité ${ }^{17}$. À l'époque moderne, la supercherie peut donc s'installer malgré l'auteur, presque à son insu, simplement - comme l'a postulé Roland Barthes - en raison de la "fatalité du signe littéraire, qui fait qu'un écrivain ne peut tracer un mot sans prendre la pose particulière d'un langage démodé, anarchique ou imité, de toutes manières conventionnel et inhumain"18. Dorénavant l'imitation, la copie, le cliché, l'épigone, l'idée reçue ou le kitsch sont stigmatisés comme des mensonges esthétiques qui constituent la cible des aspirations modernes à une véritable originalité.

\section{Entre mensonge esthétique et impératif d'authenticité : le dilemme de la littérature postmoderne}

Devient dès lors mensonger tout roman qui n'est pas capable - comme l'écrit Vargas Llosa dans La vérité par le mensonge - de faire adhérer son lecteur au monde fictif qu'il crée. Évidemment, il s'agit là d'un mensonge au second degré qui se greffe à l'intérieur du premier "mensonge" établi par la fictionnalité de l'histoire inventée. Vargas Llosa constate: "Tout bon roman dit la vérité et tout mauvais roman ment, car 'dire la vérité', pour un roman, c'est arriver à faire vivre au lecteur une illusion, et 'mentir', c'est ne pas se montrer à la hauteur de cette supercherie"19. Ce mensonge spécifique se traduit sur le plan esthétique par des clichés, des stéréotypes, des personnages et histoires convenus ou toutes les formes du kitsch. L'exemple des romans sentimentaux de Delly illustre sans doute à merveille cette forme spécifique de mensonge dans la mesure où ils entreprennent de créer des personnages extraordinaires qui se meuvent dans un contexte dont le roman fait valoir l'excellence. Mais l'écriture produit souvent un effet contraire en surchargeant les descriptions d'épithètes hyperboliques qui finissent par rendre, à coup de répétitions à outrance, l'extraordinaire ordinaire. Nous avons donc une divergence entre conviction (le monde fictionnel) et expression (la forme littéraire), une divergence que Delly tente justement de camoufler. Il en va de même pour tous les types d'imitations épigonales, pour les reproductions de formes 
jadis originales, pour les formules toutes trouvées ou pour les genres qui épousent un schéma stéréotypé comme le roman d'amour, d'aventures ou bien policier. condition postmoderne qui l'empêche d'y répondre? C'est ce dilemme qui peut expliquer une partie des orientations, des choix d'écriture, des moyens et des objectifs de la littérature d'aujourd'hui. au réel qui caractérise la littérature contemporine. Enqueter, collecter, documenter, archiver : ces gestes sont en effet devenus ceux par lesquels la littérature s'ancre dans le monde ${ }^{22}$ et tente de répondre à sa quête d'authenticité. Docufiction, factographie, non-fiction, enquêtes, autofiction, littératures de terrain: ces approches ont en commun de déplacer, pour la dépasser, la question de l'authenticité des formes littéraires et du langage vers la référence au monde réel ou social. Même si le statut de ces récits oscille entre pacte factuel et pacte fictionnel, force est de constater qu'ils puisent une certaine authenticité - et de fait une certaine légitimité - dans leur référence au réel. Emmanuel Carrère ne laisse par exemple aucun doute à ce sujet : "J'ai une conviction, une seule, concernant la littérature, enfin le genre de littérature que je pratique : c'est le lieu où on ne ment pas. C'est l'impératif absolu, tout le reste est accessoire, et à cet impératif je pense m'être toujours tenu. Ce que j'écris est peut-être narcissique et vain mais je ne mens pas"23.

Mais par un surprenant retour de manivelle, ce pas de côté fait que la littérature de l'extrême contemporain devient - et ceci constitue une nouveauté - capable de mentir. Une nouvelle "ère du mensonge" s'ouvre avec l'ère du réel. Preuve s'il en est: la 
multiplication des procès intentés suite à la parution de livres auto- ou docufictionnels. Christine Angot a ainsi été condamnée pour atteinte à la vie privée pour son roman Les petits qui décrit l'ex-femme et les enfants de son concubin ${ }^{24}$. Si le cas Angot indique simplement qu'il y une part factuelle dans l'autofiction, d'autres œuvres sont impliquées dans des polémiques autour du caractère véridique ou mensonger de leurs affirmations. C'est à cause du pacte de véracité évoqué par Emmanuel Carrère que son ex-femme, Hélène Devynck, peut pointer du doigt les mensonges qui jalonneraient Yoga (2020). Les mots qu'elle écrit sont révélateurs: "Mais au-delà, l'autre raison pour laquelle je ne voulais pas être dans ce livre, c'est l'effacement de la frontière entre fiction et mensonges. La fiction veut dire une vérité. Le mensonge veut la dissimuler" 25 . Des accusations semblables ont été prononcées à l'encontre d'Edouard Louis pour L'histoire de la violence, Raphaël Enthoven pour Le temps gagné et Yann Moix pour Orléans. Plus récemment, c'est Thésée, ma vie nouvelle de Camille de Toledo qui s'est vu discrédité au motif de sa soi-disant inexactitude factuelle et biographique ${ }^{26}$. Une telle liste est loin d'être exhaustive ou close, mais elle souligne à quel point, dans les romans autofictionnels, un pacte factuel est toujours à l'œuvre, rappelant la prétention à l'authenticité et suscitant des soupçons quant à un contenu potentiellement mensonger. Tandis que le pacte fictionnel relègue celui-ci au rang de mensonge au second degré (c'est-à-dire à un mensonge à l'intérieur du "mensonge" censément fictionnel - qui n'en est pas un), l'autofiction et la docufiction sont, elles, capables de mentir au premier degré. À cette première forme de mensonge s'en ajoute un deuxième, plus profond, qui concerne l'ambiguïté de l'instance énonciatrice et de l'histoire racontée, car les textes en question jouent sur un double statut, fictionnel et réel. En ceci, les difficultés rencontrées avec l'autofiction portent bien au-delà, puisqu'elles innervent tout un ensemble de récits dont le fondement énonciatif peut également varier entre ethnofictions, fact-fictions ou les "factions", et littératures de terrain ${ }^{27}$.

C'est ainsi à la lumière d'une théorie du mensonge et de la mauvaise foi que le retour d'une partie de la littérature contemporaine à l'Histoire, au social ou au réel, peut être lu. Car c'est bien dans le lien qu'elles tissent avec ces derniers que les œuvres puisent aussi une certaine authenticité, laquelle les rend également à même de dire la vérité ou de mentir.

Cette situation impose, en tout état de cause, d'établir avec le réel un lien le plus direct possible. Aussi la littérarité ne doit-elle pas faire obstacle en rappelant, par ses signes, que le texte n'est que texte. C'est pourquoi nombre d'œuvres se sont efforcées d'estomper les traits les plus saillants de l'écriture, jusqu'à la rendre transparente, diaphane ou blanche. Le style paraît dès lors s'effacer au profit de la seule densité du réel, rejoignant ce que Roland Barthes avait jadis appelé le "degré zéro de l'écriture". Certes, en forgeant cette notion, Barthes visait certaines œuvres du Nouveau Roman tout comme l'écriture journalistique qui avait en partie inspiré Camus pour L'étranger. Mais du minimalisme contemporain à l'écriture plate d'Annie Ernaux, les enjeux diffèrent en partie puisqu'il s'agit bien de construire une authenticité qui paraît toujours se refuser à l'œuvre littéraire ${ }^{28}$. Avec $L a$ place par exemple, il n'en allait pas seulement, pour Ernaux, de concevoir une écriture claire et limpide, capable de représenter d'une manière non littéraire la réalité familiale et sociale. L'enjeu était aussi un acte d'engagement dans lequel le souci de transparence se fait tangible, transparence qui est également discernable chez Patrick Modiano, Yves Ravey, 
Emmanuèle Bernheim, Hélène Lenoir, Hervé Guibert, Emmanuel Carrère, ou dans les docu-fictions de François Bon, porté par l'objectif de "recourir à moins d'artifice" 29 .

\section{Pour une esthétique du mensonge}

31 Mais une deuxième porte de sortie existe pour se soustraire au dilemme entre une littérature nécessairement mensongère et l'exigence d'authenticité qui la dirige. Non pas se trouver des gages d'authenticité en dehors de la littérature, dans le réel, le sujet ou l'Histoire, mais se servir des particularités structurelles du mensonge qui font que les œuvres ne sauraient être suspectées de mentir. Une partie de la littérature contemporaine recourt alors fréquemment à ce paradoxe : annuler le mensonge en le dénonçant - et ce à l'intérieur de la fiction. De cette façon, au sein du premier mensonge révélé de la fiction, s'installe un deuxième mensonge ou un mensonge au second degré. À la différence du mensonge esthétique, ce mensonge-là est signalé par l'œuvre elle-même.

Ce phénomène n'est pas toujours aussi tonitruant que dans le cas d'un narrateur délibérément non fiable, qui ouvre les vannes à sa mauvaise foi et à ses partis pris, comme dans Veuves au maquillage de Senges ou L'œuvre posthume de Thomas Pilaster de Chevillard. Car c'est aussi en sourdine, de manière plus ou moins latente, que le mensonge peut être exhibé pour être annulé. Ainsi du réemploi très net de formes traditionnelles et peut-être obsolètes, de genres littéraires mineurs, populaires et désuets qui sont délibérément pastichés, ou encore de stéréotypes et de clichés qui mettent ouvertement en relief le caractère inauthentique des personnages ou des actions. Le mensonge esthétique est placé sous les yeux du lecteur jusqu'à être désamorcé.

Chopin, Suzy Clair, Piranese, Tarzan, Le Flétan, Gloire, Patronaz, Béliard chez Echenoz, Edmondsson ou Kovalskazinski, Jean Marie ou Kabrowinski dans La salle de bain de Toussaint, le chat Alexandre Vladimirovitch, le romancier Georges Mornacier, l'inspecteur Sheralockiszyku Holamesidjudjy dans la trilogie d'Hortense chez Jacques Roubaud: ces noms de personnages sonnent faux. Parodiques et invraisemblables, ils sont même parfois directement importés de l'univers de la consommation le plus trivial, à l'image d'un certain Javel chez Echenoz et de Gibbs qui tient à préciser en se présentant: "Gibbs, comme la famille du dentifrice, vous savez, des cousins, on ne les fréquente pas" ${ }^{\prime 30}$. Le romancier émaille de la sorte son récit d'ornements en toc à l'aide de tout un attirail issu de clichés ou de genres populaires qui, par leur caractère kitch ou comique, mettent sans cesse à distance l'histoire racontée. Dans Cherokee d'Echenoz, certains protagonistes portent par exemple une coiffure à la "Angie Dickinson dans Point Blank" 31 ou ressemblent à des acteurs de cinéma comme Edwige Feuillère, tandis que d'autres donnent l'impression d'un portrait-robot dessiné "par un homme qui voudrait décrire à la fois Michèle Morgan et Grace Kelly, cet homme étant Walt Disney"32. C'est ainsi que les œuvres exacerbent l'inauthenticité d'un personnage qui manque à dessein de densité psychologique ou existentielle.

Plus largement, ce sont volontiers les genres du passé qui font l'objet d'une reprise amusée et assumée, aussi bien chez Viel, Echenoz que chez Roubaud. Il s'agit alors de pasticher un modèle tout en exposant son inauthenticité, pour en abolir du même coup le caractère mensonger. Pareille spécificité de la littérature contemporaine apparaîtra plus nettement si l'on compare par exemple son réemploi du roman policier avec celui 
qu'avait effectué le Nouveau Roman ${ }^{33}$. Si L'emploi du temps de Butor fait dérailler l'investigation du "détective" ou si Robbe-Grillet laisse Wallas commettre par erreur le meurtre sur lequel il enquête dans Les gommes, c'est moins pour mettre en lumière l'artificialité de modèles déconstruits que pour réinstaurer une forme de récit plus authentique. Au contraire, Echenoz ou Roubaud jouent des ficelles du genre et en dénudent avec jubilation le caractère factice.

Romans d'aventures, romans à énigme ou d'espionnage, science-fiction, cinéma américain, téléfilms : tous font l'objet d'une même surenchère comique ${ }^{34}$. C'est le kitch, le trivial et le romanesque outré que ces formes introduisent dans le récit, revendiquant à divers degrés une esthétique du faux. Dans Je m'en vais par exemple, lorsque Baumgartner est sur le point d'assassiner Le Flétan en l'enfermant dans une camionnette réfrigérée, ce dernier lui objecte que le procédé est d'une banalité confondante : "on tue les gens comme ça dans tous les téléfilms, ça n'a vraiment rien d'original" 35 . Baumgarnter y consent, en ajoutant cependant: "je revendique l'influence des téléfilms. Le téléfilm est un art comme un autre" ${ }^{36}$. Une telle profession de foi ne serait certainement pas décriée par bon nombre de romanciers contemporains.

Si ces reprises sont tant le signe d'une distance ironique que d'une prise au sérieux de la légitimité de ces genres stéréotypés, c'est une attitude similaire qui se manifeste à l'égard de la littérature en général. Plus exactement, ce sont aussi les procédés caractéristiques d'une certaine modernité qui font l'objet d'un même traitement oscillant entre désinvolture et assentiment.

Ainsi en va-t-il d'un principe cardinal de la modernité littéraire : l'intertextualité. De l'allusion à la réécriture, celle-ci avait permis aux œuvres de se référer à elles-mêmes et d'affirmer leur autonomie. Rien de cet ordre chez Roubaud, Viel, Echenoz, Chevillard, Senges ou Toussaint. Lorsque, par exemple, dans La salle de bain, le narrateur reçoit une invitation de l'ambassadeur autrichien Eigenschaften, le lecteur averti ne peut qu'y voir une référence au chef d'œuvre de Robert Musil, Der Mann ohne Eigenschaften (L'homme sans qualités). Certes le récit confirme cette piste par une série d'analogies : comme le héros de Musil, Ulrich, le narrateur de Toussaint se complaît dans une pure passivité et se dévoue à ses méditations. Mais, se délestant de tout projet, il devient surtout, contrairement à ce qui se passe chez Musil, un pur observateur d'expériences sans sujet. Dès lors, L'homme sans qualités ne fonctionne plus à la manière d'un modèle à part entière pour le récit: il n'est qu'une référence qui émerge au détour d'une anecdote sans portée. L'intertextualité ne soutient plus une densité de significations qui se surajoute au texte : elle tourne à vide en se banalisant. Il en irait de même pour un autre procédé copieusement mis à profit dans les expériences autoréflexives de la modernité : la mise en abyme. Le contraste qu'on peut repérer, au sujet de la réflexion sur la pluie, entre La salle de bain et La route des Flandres de Claude Simon, est saisissant. Dans les deux textes, un passage offre une description minutieuse, en gros plan, de la pluie et de ses gouttes ${ }^{37}$. Si, chez Claude Simon, l'épisode fonctionne comme une mise en abyme de la poétique du roman tout entier, où différentes couches temporelles se superposent en une sorte de surimpression, on pourrait avoir le même sentiment chez Toussaint. Seulement, le narrateur achève le passage sur ce cri : "Olé". Signe d'une désinvolture et d'un refus de se prendre au sérieux, l'interjection dénie toute authenticité aux réflexions qui viennent d'être développées. Elle balaye leur capacité à définir l'œuvre elle-même en les réduisant à une pirouette littéraire artificielle qui ne 
vise qu'à impressionner le lecteur. Ainsi, une technique d'autoréférence autrefois destinée à transformer la "représentation" littéraire en une "présence-à-soi" se fait ici le signe de la nature précieuse et prétentieuse d'une telle auto-présence. L'esthétique de la mise en abyme devient la cible d'une mise en boîte irrévérencieuse et non d'une authenticité du récit.

\section{Pour une esthétique du mensonge}

Recyclage des formes les plus diverses, de la littérature savante à la littérature populaire en passant par le cinéma, des clichés et du kitch culturel : ces éléments disent à quel point l'inauthenticité est délibérément adoptée et calculée pour être mieux révoquée. Il ne faudrait toutefois pas en déduire qu'il s'agit ainsi de remplacer une forme particulière d'authenticité, qui a fait long feu, par une autre. Il s'agit plutôt de dire adieu une fois pour toutes à la catégorie d'authenticité elle-même. C'est de la sorte que naît une littérature du mensonge esthétique, qui regarde les réalisations de la modernité avec le même plaisir désenchanté et désintéressé que tout ce qu'Adorno et Horkheimer avaient rangé sous le nom d"'industrie culturelle". Mais un tel rejet de l'authenticité ne peut pas être réalisé à partir d'une position de supériorité : il doit s'effectuer de l'intérieur, depuis une attitude qui ne s'évertue pas à dépasser, ou périmer, les partis pris de la modernité, mais beaucoup plus modestement à les subvertir par une "sous-enchère". Partant, cette esthétique du mensonge obéit à la même loi paradoxale que celle qui prévaut pour la fiction. La revendication ouverte du mensonge esthétique, telle qu'elle est pratiquée par une partie du roman contemporain, contribue en même temps à son empêchement: elle annonce le mensonge comme pour mieux l'annuler. Il en irait de même de tous ces textes extravagants, qui désertent délibérément le récit mimétique et donnent congé à la vraisemblance, que ce soit chez Chevillard, Senges ou dans Ward de Frédéric Werst.

Comme toute littérature, la littérature de l'extrême contemporain pose donc la question de la forme esthétique et de la langue, mais elle met en scène le problème central de l'expression d'une manière bien différente de celle de ses prédécesseurs. Alors que ceux-ci détruisaient les lieux communs, les formules toutes trouvées, les clichés et stéréotypes, les auteurs de l'extrême contemporain préfèrent parfois figurer l'indisponibilité d'un nouveau langage et l'impossibilité de créer de nouvelles formes d'expression aptes à traduire les pensées et les sentiments. De cette condition postmoderne, ils tirent une conséquence: celle de mettre en scène des mensonges esthétiques et d'exposer ceux-ci afin de désigner, par cette négativité, une forme authentique qui n'est plus à leur disposition. 


\section{NOTES DE FIN}

1. Voir notamment Dominique Viart, "Mémoires du récit : questions à la modernité", in Dominique Vart (dir.), Écritures contemporaines, 1, Paris-Caen, Lettres modernes Minard, 1998, <La revue des lettres modernes>, p. 3-27.

2. Platon, La république, traduction nouvelle avec introduction et notes par Robert Baccou, in Euvres complètes, vol. IV, Paris, Garnier, 1950, Livre VII, 514a, p. 247sq.

3. Ibid., (378b), p. 69. La condamnation définitive elle-même apparaît sous la forme d'une fausse question, rhétorique, dans ce dialogue également toujours faux, car maïeutique : “C'est pourquoi il importe extrêmement que les premières choses qu'il [i.e. le jeune homme] entendra soient des fables les plus propres à le porter à la vertu."

4. Ibid., (377e), p.68sq.

5. Le noble mensonge de Socrate ramène les différences entre les groupes hiérarchiques de l'État, à savoir les philosophes, les gardiens et les paysans non pas au résultat de l'éducation, mais à des qualités innées dues à la différence entre les métaux plus ou moins précieux que Dieu leur a octroyés: de l'or pour les philosophes ou dirigeants de l'État, de l'argent pour les gardiens et du fer ou du bronze pour les paysans (ibid., 414c, p. 117sq.).

6. Cf. Jochen Mecke, "Une critique du mensonge par-delà le bien et le mal", Cahiers d'Études Germaniques, n67, 2014, p. 98.

7. "L'art traite l'apparence comme une apparence, il ne veut donc pas tromper, il est vrai." (Friedrich Nietzsche, "Nachlass 1869-1874", in Kritische Studienausgabe, éd. Giorgio Colli et Mario Montinari, München, dtv, 1999, p. 632 sq.).

8. Voir Jean-François Jeandillou, Esthétique de la mystification. Tactique et stratégie littéraires, Paris, Minuit, 1994, <Propositions>.

9. Voir Wayne C. Booth, The Rhetoric of Fiction, Chicago, University of Chicago Press, 1961 ; Frank Wagner, "Quand le narrateur boit(e)... (Réflexions sur le narrateur non fiable et/ou indigne de confiance)", Arborescences, $\mathrm{n}^{\circ}$ 6, septembre 2016, disponible sur <https://id.erudit.org/iderudit/ 1037508ar>.

10. Cf. Jochen Mecke, "Die Atopie des Pícaro: Paradoxale Kritik und dezentrierte Subjektivität im Lazarillo de Tormes", in Wolfgang Matzat, Bernd Teuber (éds), Welterfahrung - Selbsterfahrung. Konstitution und Verhandlung von Subjektivität in der spanischen Literatur der frühen Neuzeit, Tübingen, Niemeyer, 2000, p. 67-94 ; Ansgar F. Nünning, "Unreliable, Compared to What? Towards a Cognitive Theory of Unreliable Narration: Prolegomena and Hypotheses", in Walter Grünzweig, Andreas Solbach (dirs), Grenzüberschreitungen : Narratologie im Kontext / Transcending Boundaries: Narratology in Context, Tübingen, Gunter Narr Verlag, 1999, p. 53-73, traduit dans Sylvie Patron (dir.), Introduction à la narratologie postclassique, Villeneuve d'Ascq, Presses Universitaires du Septentrion, 2018, p. 121-146.

11. Voir Maxime Decout, En toute mauvaise foi, Paris, Minuit, 2015, <Paradoxe>.

12. Nathalie Sarraute, L'ère du soupçon, Euvres complètes, Paris, Gallimard, 1996, <La pléiade>, p. 1554.

13. Ibid.p. 1554.

14. Friedrich Nietzsche, "Über Wahrheit und Lüge im außermoralischen Sinn", in Friedrich Nietzsche, Werke, Band III, Karl Schlechta (ed.), Frankfurt, Ullstein, 1972, p. 314, traduction française dans Friedrich Nietzsche, Le livre du Philosophe, traduit par Angèle Kremer-Marietti, Paris, édition Aubier-Flammarion, 1969.

15. Anne Villelaur, "Le nouveau roman est en train de réfléchir sur lui-même", Les Lettres françaises, $\mathrm{n}^{\circ} 764,12-18.03 .1959, \mathrm{p} .1$. 
16. Charles Baudelaire, Le peintre de la vie moderne, in Euvres, Paris, Seuil, 1968, < L'Intégrale>, p. 553.

17. Cf. Jochen Mecke, "Der Prozess der Authentizität”, in Susanne Knaller, Harro Müller (dirs), Authentizität, Paderborn, Fink, 2006, p. 82-114.

18. Roland Barthes, Le degré zéro de la littérature suivi de Nouveaux essais critiques, Paris, Seuil, 1972, $<$ Points>, p. 62.

19. “Toda buena novela dice la verdad y toda mala novela miente. Porque 'decir la verdad' para una novela significa hacer vivir al lector una ilusión y 'mentir' ser incapaz de lograr esa superchería." (Mario Vargas Llosa, La verdad de las mentiras: ensayos sobre literatura, Barcelona, Seix Barral, 1990, p. 10).

20. Theodor W. Adorno, Ästhetische Theorie, Frankfurt, Suhrkamp, 1970, p. 61sq.

21. Le rapport de la littérature à l'authenticité a été marqué par plusieurs crises, notamment au $\mathrm{XVIII}^{\mathrm{e}}$ siècle et à la fin du XIX ${ }^{\mathrm{e}}$ siècle, qui ont été déterminantes dans l'évolution des formes et des enjeux des textes (voir, notamment, Maxime Decout, En toute mauvaise foi, op. cit., et Qui a peur de l'imitation?, Paris, Minuit, 2017, <Paradoxe>).

22. Voir notamment Laurent Demanze, Un nouvel âge de l'enquête, Paris, Corti, 2019, <Les essais>.

23. Jean-Claude Vantroyen, "La littérature, le lieu où l'on ne ment pas", Le Soir, 29/08/2020.

24. Pascale Robert-Diard, "Le jugement qui condamne Christine Angot pour atteinte à la vie privée", Le Monde, 28/05/2013, disponible sur <https://www.lemonde.fr/justice/article/ 2013/05/28/le-jugement-qui-condamne-christine-angot-pour-atteinte-a-la-vieprivee_6002297_1653604.html>.

25. Hélène Devynck, "Droit de réponse", Vanity Fair, 29/09/2020, disponible sur <https:// www.vanityfair.fr/culture/voir-lire/articles/droit-de-reponse-helene-devynck-l-ex-compagnedemmanuel-carrere-repond-a-la-polemique-autour-de-yoga/81120>.

26. Claire Paulhian, "La fabrique d'une légende", En attendant Nadeau, 18 novembre 2020, disponible sur <https://www.en-attendant-nadeau.fr/2020/11/18/fabrique-legende-toledo/>.

27. Voir notamment Jean-Louis Jeannelle, "Histoire littéraire et genres factuels", Fabula-LhT, n zéro, "Théorie et histoire littéraire", février 2005, disponible sur <http://www.fabula.org/lht/0/ jeannelle.html>, page consultée le 10 juin 2019. Pour la littérature de terrain, voir Dominique Viart, "Les Littératures de terrain", Revue critique de fixxion française contemporaine, $\mathrm{n}^{\circ} 18,2019, \mathrm{p}$. 1-13, ainsi que le numéro entier de la revue consacré à ce genre relativement nouveau.

28. Voir entre autres à ce sujet Warren Motte, Small Worlds: Minimalism in Contemporary French Literature, Lincoln, University of Nebraska Press, 1999 ; Marc Dambre, Bruno Blanckeman (dirs), Romanciers minimalistes. 1979-2003, Paris, Presses Sorbonne Nouvelle, 2012, <Fiction/Non fiction XXI>.

29. Francois Bon, Dominique Viart, “On écrit avec de soi” [entretien], Revue des sciences humaines, $\mathrm{n}^{\circ} 263$ (juillet-septembre), 2001, p. 61.

30. Jean Echenoz, Cherokee, Paris, Minuit, p. 117.

31. Ibid., p. 61.

32. Ibid., p. 28.

33. Voir Maxime Decout, Pouvoirs de l'imposture, Paris, Minuit, 2018, <Paradoxe>,.

34. Voir notamment Simon Kemp, Detective Inspectors: Crime-fiction Pastiche in Late Twentiethcentury French Literature, Oxford, Legenda, 2006 ; Bruno BLANCKEMAN, “Jean Echenoz ou le roman comme ciné/cure", Roman 20-50, vol. 41, n 1, 2006, p. 167-178.

35. Jean Echenoz, Je m'en vais, Paris, Minuit, 1999, p. 152

36. Ibid.

37. Claude Simon, La route des Flandres, Paris, Minuit, , 1982 [1960], <double>, p. 24 ; Jean-Philippe Toussaint, La salle de bain, Paris, Minuit, p. 35s. 
AUTEURS

MAXIME DECOUT

Aix-Marseille université - IUF

JOCHEN MECKE

Université de Regensburg 